\title{
Estilo de vida e consumo de alimentos funcionais em docentes do Ensino Superior
}

Lifestyle and functional food consumption in Higher Education Teachers

\author{
Cati Reckelberg Azambuja', Daniela Lopes Santos", \\ Andressa da Rosa Rodrigues'", Vanessa Albanio Machadoiv, \\ Anaelena Bragança de Moraes $v$
}

\begin{abstract}
RESUMO
O objetivo foi analisar o estilo de vida e o consumo de alimentos funcionais de docentes de uma Instituição Federal de Ensino Superior do sul do Brasil. Foi solicitado os endereços eletrônicos dos docentes e enviado a todos os 1823 um convite via e-mail, junto às explicações da pesquisa. A participação ocorreu pelo preenchimento online (Enquete Fácil), do "Questionário Estilo de Vida Fantástico", traduzido e validado para o Brasil e o Questionário de Consumo alimentar de autoria própria, contendo 16 grupos de alimentos funcionais. Dos 1823 docentes da instituição, 280 participaram do estudo, sendo 134 homens e 146 mulheres, com média de idade de 46,3 anos (desvio padrão 9,2). A média dos escores do estilo de vida foi 73,8 e para o consumo de alimentos funcionais foi de 9,4 grupos de alimentos (desvio padrão 3,0). Os escores do estilo de vida apresentaram correlação significativa com o consumo de alimentos funcionais, tanto na amostra total $(p<0,001)$, como na estratificada por sexo $(p<0,05)$. Já a idade apresentou correlação significativa com o estilo de vida somente para os homens $(p=0,004)$. Portanto, os docentes apresentaram um estilo de vida considerado muito bom, bem como um bom consumo de alimentos funcionais, e quanto melhor o estilo de vida destes, maior a ingestão de alimentos funcionais.
\end{abstract}

Palavras-chave: Comportamento saudável. Nutrição. Universidade.

\begin{abstract}
The purpose was to aanalyse the lifestyle and functional foods' consumption among faculty members of a Federal Institution of Higher Education in southern Brazil. The Dean of the Personnel Department informed the electronic addresses of the 1823 Institution professors, and an email was sent to each of them inviting to participate, along with the explanations about the research. Participants had to fill online the "Lifestyle Fantastic Questionnaire", which was translated and validated for Brazil and a food frequency questionnaire, prepared by the authors, containing 16 functional food groups. From the total number of 1823 institution professors, 280 participated in the study, 134 were men and 146 were women, with a mean age of $46.3 \pm 9.2$ years. The average lifestyle score was $73.8 \pm 11.6$ points and the consumption of functional foods was of $9.4 \pm 3.0$ types of foods. The lifestyle score was significantly correlated with the consumption of functional foods, both in the total sample, as when the sample was divided by sex $(p<0.001 ; p=0.025)$. In contrast, age was significantly correlated with the lifestyle score only in men $(p=0.004)$. Therefore, the professors presented a lifestyle considered very good and also a good consumption of functional foods. Besides, it was also concluded that the better the lifestyle of the studied sample, the greater was the intake of functional foods.
\end{abstract}

Keywords: Health behavior. Nutrition. University.

'Universidade Federal de Santa Maria, Brasil; e-mail: cati.razambuja@hotmail.com;

" Universidade Federal de Santa Maria. Brasil. e-mail: lopesdossantos@gmail.com;

III Universidade Federal de Santa Maria, Brasil; e-mail: nutricionistaandressa@hotmail.com;

Iv Universidade Federal de Santa Maria. Brasil. e-mail: vanessaalbanio@gmail.com;

v Universidade Federal de Santa Maria. Brasil. e-mail: anaelena.bm@terra.com.br 


\section{INTRODUÇÃO}

"Alimentos funcionais são aqueles que produzem efeitos fisiológicos ou metabólicos, por meio do desempenho de algum nutriente, na manutenção das funções do organismo humano" (VIDAL et al., 2012, p. 44). Considera-se um alimento como funcional se o mesmo demonstrar que pode afetar beneficamente uma ou mais funções alvo no corpo, além de possuir os adequados efeitos nutricionais, de maneira que seja tanto relevante para o bem-estar e a saúde quanto para a redução do risco de uma doença (ROBERFROID, 2002). Podem ser classificados de dois modos: quanto à fonte, de origem vegetal ou animal, ou quanto aos benefícios que oferecem, atuando em seis áreas do organismo: no sistema gastrointestinal; no sistema cardiovascular; no metabolismo de substratos; no crescimento, no desenvolvimento e diferenciação celular; no comportamento das funções fisiológicas e como antioxidantes (SOUZA, SOUZA NETO e MAIA, 2003). Esses alimentos funcionais auxiliam na prevenção e no tratamento de algumas doenças, ajudando o organismo a combatê-las de maneira mais eficaz. Para tanto, devem ser incorporados numa alimentação para que possam ser consumidos diariamente, ajudando o organismo a se fortalecer. Uma dieta rica em alimentos funcionais acarreta um maior bem-estar, dando mais disposição e energia para os que a consomem, contribuindo assim, para uma melhoria da qualidade de vida (VIDAL et al., 2012).

Nahas, Barros e Francalacci (2000) relatam que há um grande impacto dos hábitos pessoais no estilo de vida e na saúde das pessoas. Sabe-se que a genética, o ambiente e a assistência médica têm um papel importante em como e quanto se vive, porém, cada vez mais crescem as evidências de que o modo de viver representa o fator diferencial para a saúde e a qualidade de vida no mundo contemporâneo, independente da idade ou condição social. Existem diversos fatores que podem afetar o estilo de vida, de forma negativa, trazendo riscos à saúde. Aqueles aos quais se pode ter controle são chamados de fatores negativos modificáveis como, por exemplo, sedentarismo, fumo, álcool em excesso, uso de drogas e a forma como se lida com o estresse. Há ainda os fatores positivos, que se ministrados de forma correta, 
contribuem também para um estilo de vida saudável, como alimentação adequada, prática regular de atividade física e comportamento preventivo.

O estilo de vida é o conjunto de ações habituais que refletem as atitudes, os valores e as oportunidades na vida das pessoas, podendo ser considerado saudável quando contribui para adicionar anos à vida e vida aos anos, auxiliando na redução das chances de aparição de enfermidades e incapacidades em indivíduos, grupos e comunidades (NAHAS, 2010; LEMOS, NASCIMENTO e BORGATTO, 2007; MADUREIRA, FONSECA e MAIA, 2003). A sociedade moderna mostra-se cada vez mais complexa, modificando os padrões de vida. Frequentemente, as pessoas têm mostrado sintomas de cansaço, depressão e irritação, ou mais comumente uma forma de estresse (KWAK e JUKES, 2001). Entretanto, a baixa incidência de doenças em alguns povos chamou a atenção para a sua dieta. Os esquimós, com sua alimentação baseada em peixes e produtos do mar ricos em ácidos graxos poliinsaturados das famílias ômega 3 e 6, têm baixo índice de problemas cardíacos, assim como os franceses, devido ao consumo de vinho tinto, o qual apresenta grande quantidade de compostos fenólicos. Já, os orientais devido ao consumo de soja, fonte de fitoestrogênios, apresentam baixa incidência de câncer de mama. Nestes países, o costume de consumir frutas e verduras também resulta numa redução do risco de doenças coronarianas e de câncer, comprovada por dados epidemiológicos (ANJO, 2004).

Por conta disso, os brasileiros começaram a adotar hábitos mais saudáveis e passaram a ingerir uma maior quantidade de frutas, grãos integrais, peixes, aves e legumes, deixando um pouco de lado as gorduras, as frituras e a carne vermelha. Assim, no decorrer das mudanças de hábitos, os chamados alimentos funcionais passaram a ser integrados a uma alimentação balanceada e consumidos de maneira correta. Assim, o objetivo deste estudo foi avaliar e relacionar o estilo de vida e o consumo de alimentos funcionais em docentes de uma Instituição Federal de Ensino Superior do Sul do Brasil. 


\section{MÉTODOS}

A população estudada foi a dos docentes de uma Universidade Federal localizada na Região Sul do Brasil. A amostra foi calculada (TRIOLA, 2013), de forma a ser representativa da população do estudo. Considerando-se que a universidade possuía um número total de 1823 docentes (número fornecido pela Pró-Reitoria de Recursos Humanos da Instituição, em dezembro de 2013), foi estimada a participação de 260 sujeitos. Como critério de inclusão, foi considerado que o docente deveria possuir email cadastrado junto à Pró-Reitoria de Recursos Humanos da Universidade, que forneceu os endereços eletrônicos aos pesquisadores. O preenchimento inadequado do questionário (online) foi adotado como critério de exclusão. Considerou-se como "recusas" todos os docentes que receberam convite para participar, via e-mail, mas não o responderam.

Tendo-se em mãos todos os endereços eletrônicos dos docentes da Universidade fornecidos pela Pró-Reitoria de Recursos Humanos, foi enviado um convite de participação via e-mail para cada um dos 1823 docentes, junto às explicações de como seria o estudo e também o Termo de Consentimento Livre e Esclarecido (TCLE), ficando explícito que ao responderem os instrumentos de coletas de dados online através do link disponibilizado, estes concordariam em participar do estudo, não sendo necessária a assinatura do TCLE. Aceitando participar, o docente deveria "clicar" no link disponibilizado no site "Enquete Fácil" para o preenchimento online dos instrumentos de coleta de dados. O site "Enquete Fácil" é uma plataforma na qual se podem inserir questionários que são disponibilizados por convite, geralmente utilizados para um grande número de pessoas.

O instrumento utilizado para averiguar o estilo de vida, foi o "Questionário Estilo de Vida Fantástico - QEVF" proposto pela Sociedade Canadense de Fisiologia do Exercício em 1998, traduzido e validado para o Brasil (RODRIGUEZ-AÑEZ, REIS e PETROSKI, 2008). O questionário possui vinte e cinco questões divididas em nove domínios: 1) Família e amigos; 2) Atividade Física; 3) Nutrição; 4) Tabaco e tóxicos; 5) Álcool; 6) Sono, cinto de segurança, estresse e sexo seguro; 7) Tipo de comportamento; 8) Introspecção; 9) Trabalho. Trata-se de um instrumento auto aplicado que permite 
determinar a associação entre o estilo de vida e a saúde através de escala do tipo Likert pontuadas conforme o valor de cada coluna, sendo: 0 (zero) para a primeira coluna; 1 (um) para a segunda coluna; 2 (dois) para a terceira coluna; 3 (três) para a quarta coluna; 4 (quatro) para a quinta coluna. Para questões binárias, a pontuação é 0 (zero) para a primeira coluna e 4 (quatro) para a última coluna. Ao final do questionário, a soma de todas as pontuações permite que os docentes sejam classificados em cinco categorias de estilo de vida: a) Excelente (85 a 100 pontos); b) Muito bom (70 a 84 pontos); c) Bom (55 a 69 pontos); d) Regular (35 a 54 pontos); e) Necessita melhorar (0 a 34 pontos). Para investigar o consumo de alimentos funcionais, foi elaborado pelas autoras um Questionário de Consumo Alimentar de Alimentos Funcionais - QCAAF (ANEXO A), baseado em evidências (MONTEIRO e MARIN, 2010; MORAES e COLLA, 2006; HASLER, 1998) contendo dezesseis grupos de alimentos com compostos funcionais: 1) Isoflavonas; 2) Ômega-3; 3) Ácido linolênico; 4) Catequinas; 5) Licopeno; 6) Luteína e Zeaxantina; 7) Indois e Isoiticianatos; 8) Flavonoides; 9) Fibras solúveis e insolúveis; 10) Prebióticos; 11) Sulfetos alílicos; 12) Lignanas; 13) Tanino; 14) Estanóis e esteróis vegetais, 15) Probióticos; 16) Betacaroteno, no qual os participantes deveriam indicar quais destes grupos de alimentos eles costumavam ingerir regularmente, podendo marcar mais de uma resposta, conforme seu consumo.

A análise dos dados foi realizada, inicialmente, pela estatística descritiva. Para as inferências, foi verificada a normalidade dos dados pelo teste de Kolmogorov-Smirnov. Utilizou-se o teste de Kruskal-Wallis e post hoc de Dunn's e o teste de correlação de Pearson para determinar o grau de correlação (DEVORE, 2006) entre a pontuação relativa ao estilo de vida e a quantidade de alimentos funcional consumida regularmente pelos docentes. O nível de significância adotado foi de $5 \%$ e os dados foram analisados com o auxílio do aplicativo computacional SPSS (Statistical Package for Social Sciences) versão 20.0. Este estudo foi aprovado pelo Comitê de Ética em Pesquisa com Seres Humanos da Universidade Federal de Santa Maria, processo $n^{\circ}$ 31303214.6.0000.5346 e parecer $n^{\circ}$ 689.488, conforme a Resolução $n^{\circ} 466 / 12$ do Conselho Nacional de Saúde (CNS/MS), que regulamenta a pesquisa com seres humanos. 


\section{RESULTADOS}

Dos 1823 docentes convidados a participar do estudo, 280 (média de idade $46,3 \pm 9,2$ anos) responderam os instrumentos online após estes terem sido disponibilizados no site de coleta de dados pelo período de 90 dias, no ano de 2014. A comparação entre os professores do sexo masculino e feminino não apresentou diferença significativa na idade e, também na pontuação total referente ao estilo de vida e a quantidade de tipos de alimentos funcionais ingeridos pelos docentes. Contudo, quando os domínios do estilo de vida foram comparados por sexo, foi possível verificar diferença significativa em dois domínios do perfil do estilo de vida (Tabela 1). Já, na Tabela 2, os docentes foram classificados conforme o nível do estilo de vida que apresentaram.

Tabela 1 - Comparação entre os sexos da pontuação média dos domínios do perfil de estilo vida dos docentes de uma Universidade Federal do Sul do Brasil.

\begin{tabular}{lccccc}
\hline Perfil do Estilo de Vida & \multicolumn{3}{c}{ Feminino $(\mathrm{n}=146 ; 52,1 \%)$} & \multicolumn{3}{c}{ Masculino (n=134; 47,9\%) } & \multirow{2}{*}{ p-valor* } \\
\cline { 2 - 5 } (domínios) & $\mathrm{X}$ & $\mathrm{S}$ & $\mathrm{X}$ & $\mathrm{S}$ & \\
\hline 1) Família e amigos & 3,45 & 0,84 & 3,30 & 0,90 & 0,637 \\
2) Atividade Física & 1,67 & 1,18 & 1,72 & 1,19 & $>0,99$ \\
3) Nutrição & 2,86 & 1,31 & 2,66 & 1,33 & 0,114 \\
4) Tabaco e tóxicos & 3,39 & 0,99 & 3,54 & 0,81 & 0,403 \\
5) Álcool & 3,57 & 0,99 & 3,20 & 1,28 & $<0,001$ \\
6) Sono, cinto de segurança, & 3,02 & 1,15 & 3,21 & 1,03 & 0,037 \\
estresse e sexo seguro & & & & & \\
7) Tipo de comportamento & 2,56 & 1,16 & 2,65 & 1,05 & $>0,99$ \\
8) Introspecção & 2,42 & 1,03 & 2,58 & 0,98 & $>0,99$ \\
9) Trabalho & 3,27 & 0,97 & 3,29 & 1,00 & $>0,99$ \\
\hline
\end{tabular}

Fonte: Elaborado pelos autores. Legenda: X: média; S: desvio padrão; Teste de: Kruskal-Wallis; *p-valor: nível de significância = 5\%.

Tabela 2 - Classificação do perfil do estilo de vida dos docentes de uma Universidade Federal do Sul do Brasil.

\begin{tabular}{lcccc}
\hline Perfil do Estilo de Vida & \multicolumn{2}{c}{ Feminino $(\mathrm{n}=146 ; 52,1 \%)$} & \multicolumn{2}{c}{ Masculino $(\mathrm{n}=134 ; 47,9 \%)$} \\
\cline { 2 - 5 } (classificação) & $\mathrm{f}_{\mathrm{i}}$ & $\mathrm{f}_{\mathrm{ri}} \%$ & $\mathrm{f}$ & $\mathrm{f}_{\mathrm{ri}} \%$ \\
\hline Necessita melhorar (0 a 34 pontos) & 2 & 1,4 & 1 & 0,7 \\
Regular (35 a 54 pontos) & 2 & 1,4 & 2 & 1,5 \\
Bom (55 a 69 pontos) & 36 & 24,7 & 38 & 28,4 \\
Muito bom (70 a 84 pontos) & 88 & 60,3 & 74 & 55,2 \\
Excelente (85 a 100 pontos) & 18 & 12,3 & 19 & 14,2 \\
\hline Total & 146 & 100,0 & 134 & 100,0 \\
\hline
\end{tabular}

Fonte: Elaborado pelos autores. Legenda: $\mathrm{f}_{\mathrm{i}}$ : frequência absoluta; $\mathrm{f}_{\mathrm{ri}} \%$ : frequência relativa percentual. 
A pontuação média do QEVF, que foi utilizada para avaliar o estilo de vida dos docentes, foi de 73,8 pontos $( \pm 11,6)$, de um máximo de 100 pontos, classificando-os com estilo de vida muito bom. Quando estratificados por sexo, tanto as mulheres quanto os homens apresentaram prevalência na mesma faixa de classificação (muito bom). $O$ consumo de alimentos funcionais pelos docentes também alcançou resultado positivo, pois de um total de 16 grupos de compostos funcionais, a quantidade média de alimentos funcionais consumidos regularmente foi de 9,4 grupos $( \pm 3,0)$ sendo que as fibras solúveis e insolúveis aparecem como prevalentes na preferência dos docentes (Figura 1).

Figura 1 - Grupos de alimentos funcionais consumidos regularmente pelos docentes de uma Universidade Federal do Sul do Brasil.

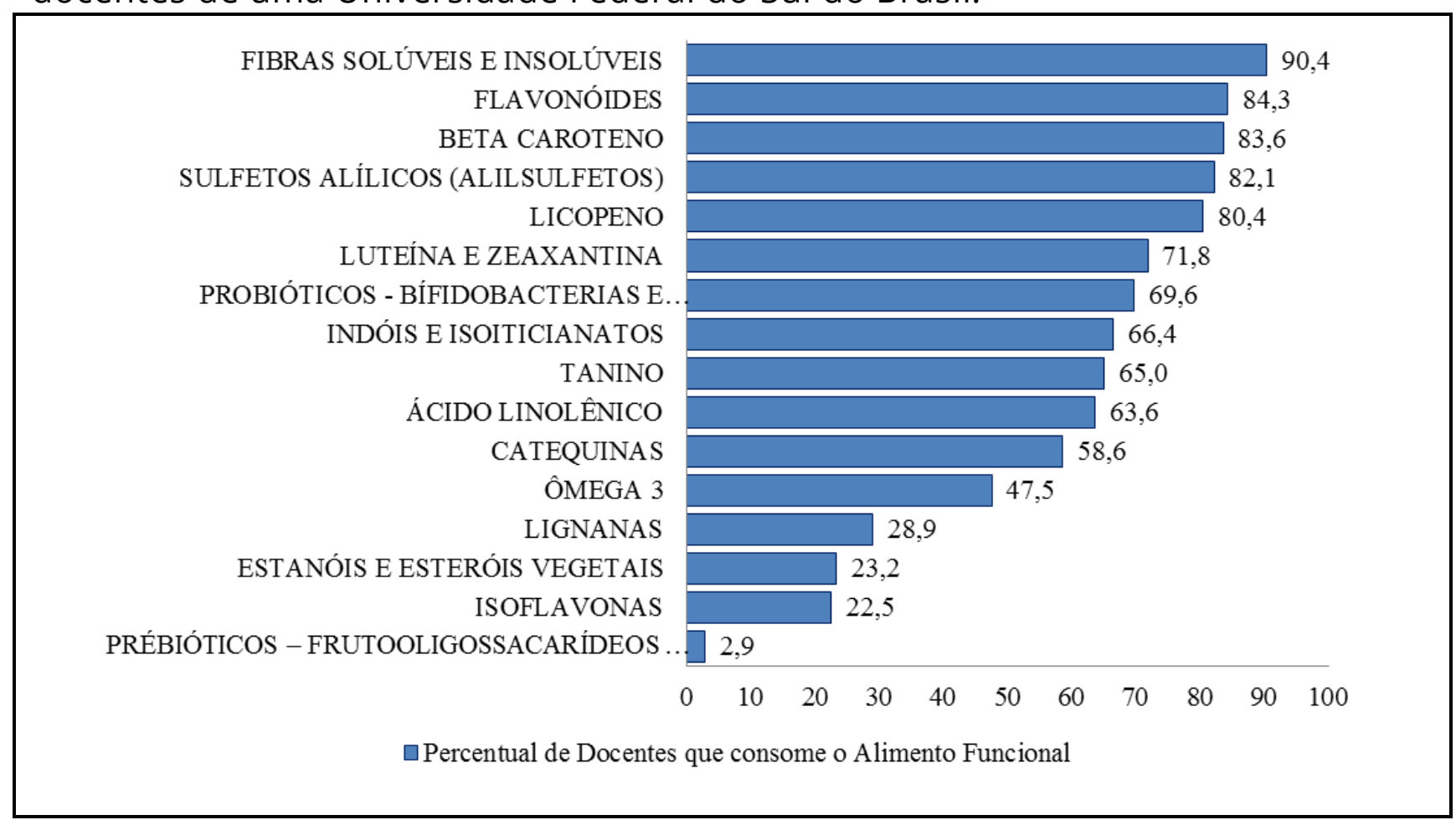

Fonte: Elaborado pelos autores.

Ao analisar a correlação entre o estilo de vida e o consumo de alimentos funcionais dos docentes, obteve-se um coeficiente de correlação significativo de magnitude moderada $(r=0,421 ; p<0,001)$, indicando que quanto melhor o estilo de vida, maior a variedade de grupos de alimentos funcionais consumidos. Porém, ao correlacionar as variáveis estilo de vida e quantidade de grupos de alimentos funcionais consumidos, estratificando-os pelo sexo, a correlação no sexo feminino foi semelhante 
a geral e a do sexo masculino mostrou-se fraca (mulheres: $r=0,449, p<0,001$; homens: $r=0,194, p=0,025)$. Já com relação à idade e ao estilo de vida, a correlação foi positiva e significativa, porém fraca e, constatada apenas nos homens $(r=0,231, p=0,007)$, indicando que nestes, quanto maior a idade, melhor o estilo de vida.

\section{DISCUSSÃO}

Observou-se uma proporção discretamente maior de participação das mulheres $(52,1 \%)$ em relação aos homens no presente estudo. Esse dado vai ao encontro de outros estudos, como o de Fernandes et al. (2009) que observaram a prevalência do sexo feminino no estudo e o de Santos e Marques (2013) que discutiram a evidência do sexo feminino ser maioria na profissão docente.

Quanto ao estilo de vida, os docentes foram classificados pelo QEVF na categoria muito boa. O estilo de vida é caracterizado por meio de comportamento identificáveis que podem ter um efeito profundo na saúde dos seres humanos e está relacionado com diversos aspectos que refletem as atitudes, os valores e as oportunidades na vida das pessoas (WORLD HEALTH ORGANIZATION, 1998). Os resultados são semelhantes aos encontrados por Fernandez et al. (2009) que utilizaram o mesmo instrumento para analisar o estilo de vida de professores universitários da Universidade Estadual do Sudoeste da Bahia, e verificaram que 55,3\% possuíam bom estilo de vida, enquanto $44,7 \%$ possuíam excelente estilo de vida. Outro estudo que avaliou a percepção da Qualidade de Vida no trabalho e o Perfil do Estilo de Vida dos docentes de Educação Física do estado de Santa Catarina por regiões (BOTH, NASCIMENTO e BORGATTO, 2008), constatando que os docentes das regiões Norte e Oeste do Estado e o Vale do Itajaí apresentaram os melhores Estilos de Vida. No cômputo geral, os valores positivos foram considerados satisfatórios em todas as regiões investigadas e os índices negativos não alcançaram valores representativos. Poucos estudos sobre o estilo de vida de professores universitário são descritos na literatura brasileira, o que dificultou a comparação dos resultados da presente pesquisa.

No que se refere ao consumo dos alimentos funcionais, os docentes responderam que ingerem mais da metade dos grupos de alimentos incluídos na pesquisa. O International Food Information Council - IFIC (1998) define alimentos 
funcionais como alimentos que provêm benefícios adicionais à saúde, além daqueles já ingeridos na dieta - carboidratos, proteínas, lipídios, vitaminas, minerais, fibras e água - e considerados como nutrientes tradicionais (DE ANGELIS, 2005, p.7). De acordo com a Resolução no 18, de 30 de abril de 1999 da Agência Nacional de Vigilância Sanitária - ANVISA (BRASIL, 1999), propriedade funcional é aquela relativa ao papel metabólico ou fisiológico que o nutriente ou não nutriente tem no crescimento, no desenvolvimento, na manutenção e em outras funções normais do organismo humano. Esse mesmo órgão descreve como alegação de propriedade de saúde aquela que afirma, sugere ou implica a existência de relação entre o alimento ou ingrediente e a doença ou condição relacionada à saúde. Os benefícios dos alimentos funcionais são decorrentes de vários efeitos metabólicos e fisiológicos que contribuem para um melhor desempenho do organismo do indivíduo que os ingere. Isto ocorre pelos mecanismos de ação através das vias bioquímicas e fisiológicas ou farmacológicas pelas quais uma determinada substância interage com os componentes celulares e/ou teciduais para realizar um consequente efeito biológico (VIDAL et al., 2012). Todos os alimentos funcionais do estudo possuem sua propriedade funcional específica ao qual trazem diversos benefícios à saúde quando usados rotineiramente.

Ao relacionar o estilo de vida com o consumo de alimentos funcionais dos docentes universitários, pode-se observar resultado significativo, de alta relevância ao estudo, pois mostra que quanto mais alimentos funcionais são ingeridos, melhor é o estilo de vida. No estudo de Salles et al. (2012) observou-se que 63,3\% dos professores estudados estavam classificados dentro do grupo com estilo de vida adequado. 0 estudo que verificou o estilo de vida dos professores de Educação Física ao longo da carreira docente no estado de Santa Catarina evidenciou que os professores com maior idade preocupam-se mais com a alimentação saudável, comparados aos professores mais jovens, principalmente nos comportamentos vinculados ao consumo de alimentos livres de agrotóxicos, não industrializados e com baixos níveis de calorias (BOTH, NASCIMENTO e BORGATTO, 2008). Em outro estudo, realizado por Szakály et al. (2012), os achados demonstraram que existe correlação significativa entre estilo de vida, comportamento saudável e consumo de alimentos funcionais. 
Apesar de se ter encontrado resultados que, de forma geral, evidenciam que os docentes possuem um bom estilo de vida e ingerem alimentos funcionais em boa quantidade, ressalta-se que existe a possibilidade de, justamente os que não têm tais atitudes positivas, simplesmente terem se recusado a participar da pesquisa, pois, ao responder um questionário deste tipo, é inevitável que o respondente realize uma autoanálise sobre o próprio comportamento preventivo, podendo causar certo desconforto psicológico.

Em uma revisão sistemática que avaliou as diferenças no consumo de alimentos funcionais em nível mundial, indicou que o sexo, a idade, o nível educacional e o status de saúde podem, cada um a sua maneira, predizer e influenciar o consumo de alimentos funcionais (OZEN, PONS e TUR, 2014). Os docentes universitários do sexo masculino, deste estudo, apresentaram correlação entre a idade e o estilo de vida, revelando que quanto maior a idade dos professores, melhor o estilo de vida. Contudo, outra pesquisa realizada com 76 docentes de uma Universidade Baiana, não encontrou a relação entre as variáveis estilo de vida e idade. Os autores verificaram que houve tendência de adoção de comportamentos inadequados à saúde à proporção que se ficava mais velho, já que dos professores mais jovens (com menos de 50 anos de idade), apenas $23,1 \%$ revelaram adotar estilo de vida inadequado. Porém, no grupo de professores com idades acima de 50 anos, esta proporção cresceu consideravelmente, atingindo $47,1 \%$ dos docentes (FERNANDES et al., 2009).

Vale destacar, que nas mulheres não se percebeu correlação estatisticamente significativa entre a idade e o estilo de vida. Talvez, este resultado se justifique devido ao período em que a mulher se encontra, em torno de 45,6 anos, pois é nessa fase que as mulheres passam pela transição do climatério, antes de chegar à menopausa. No climatério, ocorrem alterações físicas e psíquicas importantes, que prejudicam a qualidade de vida da mulher, o que pode ou não potencializar problemas de saúde (ROCHA e ROCHA, 2010). Alguns autores enfatizam que cerca de 50 a 70\% das mulheres referem sintomas somáticos e dificuldades emocionais no climatério, interferindo no seu bem-estar, estando relacionados principalmente à dificuldade de aceitar o processo de envelhecimento, simbolizado pela menopausa (SILVA-FILHO e COSTA, 
2008; LINDH-ASTRAND et al., 2007; DE LORENZI et al., 2008). Como exemplo de estudo brasileiro abordando a questão da qualidade de vida no climatério está a pesquisa realizada na Universidade de Caxias do Sul (UCS), que visou avaliar a qualidade de vida de 323 mulheres pós-menopáusicas com idade entre 45 e 60 anos. A sua análise revelou uma tendência à deterioração da qualidade de vida entre a população estudada, em particular nos domínios relacionados a sintomas somáticos, humor deprimido e ansiedade (DE LORENZI, 2008).

Outra hipótese que pode ser levantada decorrente do presente resultado é a inserção da mulher em um espaço por muito tempo considerado masculino, o que acabou por fazer com que a mulher assumisse tanto o trabalho fora de casa como o trabalho doméstico, sobrecarregando-a com uma dupla jornada de trabalho (FRANÇA e SCHIMANSKI, 2009). O contexto acadêmico, que é o foco deste estudo, parece ser um ambiente estressor por excelência. Tanto é que a docência é considerada, como uma das profissões mais estressantes (MELEIRO, 2006; SILVA, 2006). A população de professores de forma geral tem um importantíssimo papel na nossa sociedade, devendo-se atentar para os cuidados com a saúde destes, bem como se investir em prevenção de doenças crônicas não transmissíveis. Assim, é surpreendente que se encontre tão poucos estudos com esta população.

Em recente estudo, Kraus, Annunziata e Vecchio (2017), foram encontrados que o sexo, a idade e a educação diferenciaram o critério de decisão em adquirir alimentos funcionais. Mulheres, pessoas mais velhas (35 a 60 anos) e com educação universitária dão maior importância a naturalidade, valor nutricional, frescor, segurança alimentar e garantia de qualidade. Os autores afirmam, ainda, que existem diferenças claras entre homens e mulheres no campo dos componentes funcionais do alimento, que são significativamente mais importantes para as mulheres do que para os homens. Sexo, idade e educação diferenciam essencialmente as preferências por produtos base: homens jovens preferem produtos a base de carne enquanto mulheres e homens mais velhos preferem produtos a base de cereais. Consumidores mais jovens são mais abertos ao processamento alimentar de alta tecnologia. As motivações são diferenciadas por idade e sexo também: homens jovens dão menos importância a 
consequências funcionais e psicológicas como a melhora da saúde, alimentação saudável, escolha consciente e promoção da saúde, diferentemente das mulheres e homens mais velhos. As mulheres e os homens mais velhos são mais interessados na segurança da saúde e são mais responsáveis pela sua saúde.

Por fim, destaca-se que no presente estudo, foi considerado como fator limitante o fato de não se ter mensurado efetivamente nenhuma das variáveis, tendo sido estas coletadas apenas através do preenchimento de questionários, o que sabidamente implica em ter de se considerar a percepção pessoal que cada indivíduo tem de si mesmo.

\section{CONCLUSÕES}

Com relação ao estilo de vida dos docentes universitários avaliados, ficou evidente que a maior parte dos investigados apresentou um estilo de vida muito bom. Quanto ao consumo de alimentos funcionais os mesmos consomem mais da metade dos grupos funcionais apresentados no estudo, e esse resultado afirma a associação benéfica da ingestão dos alimentos funcionais em grande variedade, com um muito bom estilo de vida. O que chamou a atenção, é que quando se analisou docentes homens e mulheres de maneira isolada, verificou-se correlação entre idade e estilo de vida, somente no sexo masculino. Sabendo que a faixa etária em que os docentes se encontram está abaixo de 50 anos, observa-se uma grande mudança em ambos os sexos, mas principalmente no público feminino, devido à transição da mulher para a menopausa, e talvez esse seja o motivo de tal resultado. Porém, para que essa suposição seja afirmada, é necessário um novo estudo. Esses resultados merecem ser evidenciados na comunidade acadêmica, pois não há muitos estudos que avaliem o estilo de vida de docentes universitários. 


\section{REFERÊNCIAS}

ANJO DLC. Alimentos funcionais em angiologia e cirurgia vascular. J Vasc Bras. 2004;3(2):145-54.

BOTH J, NASCIMENTO JV, BORGATTO AF. Estilo de vida dos professores de Educação Física ao longo da carreira docente no estado de Santa Catarina. Rev Educ Fís/UEM 2008;19(3):377-89.

BRASIL. Agência Nacional de Vigilância Sanitária (ANVISA). Resolução-RDC n¹8, de 30 de abril de 1999. Aprova o regulamento técnico que estabelece as diretrizes básicas para análise e comprovação de propriedades funcionais e/ou de saúde alegadas em rotulagem de alimentos. Disponível em:

http://portal.anvisa.gov.br/wps/wcm/connect/815ada0047458a7293e3d73fbc4c6735/ RESOLUCAO_18_1999.pdf?MOD=AJPERES. Acesso em: 15set2015.

DE ANGELIS, RC. A importância dos alimentos vegetais na proteção da saúde: fisiologia da nutrição protetora e preventiva de enfermidades degenerativas. 2.ed. São Paulo: Atheneu, 2005, 317p.

DE LORENZI DRS, BARACAT EC, SACILOTO B, PADILHA JÚNIOR I. Fatores associados à qualidade de vida após menopausa. Rev Assoc Méd Bras. 2006;52(5):312-7.

DE LORENZI, DRS. Avaliação da qualidade de vida no climatério. Rev Bras Ginecol Obstet. 2008;30(3):103-6.

DEVORE, JL. Probabilidade e estatística: para engenharia e ciências. São Paulo: Pioneira Thomson Learning, 2006.

FERNANDES MH, PORTO GG, ALMEIDA LGD, ROCHA VM. Estilo de vida de professores universitários: Uma estratégia para a promoção da saúde do trabalhador. Rev Bras Prom Saúde 2009;22(2):94-9.

FRANÇA AL, SCHIMANSKI E. Mulher, trabalho e família: uma análise sobre a dupla jornada feminina e seus reflexos no âmbito familiar. Emancipação 2009;9(1):65-78.

HASLER C. Alimentos funcionais: seu papel na prevenção de doenças e na Promoção da Saúde. Braz J Food Tech. 1998;52(2):57-62.

IFICF - INTERNATIONAL FOOD INFORMATION COUNCIL FOUNDATION. Backgrounder: functional foods. In: Food insight media guide. Washington (DC): IFIC Foundation, 1998. 
KRAUS A, ANNUNZIATA A, VECCHIO R. Sociodemographic Factors Differentiating the Consumer and the Motivations for Functional Food Consumption. J Am Coll Nutr. 2017;9:1-11.

KWAK N, JUKES DJ. Functional foods - part 1: the development of a regulatory concept. Food Control 2001;12:99-107.

LEMOS CAF, NASCIMENTO JV, BORGATTO AF. Parâmetros individuais e socioambientais da qualidade de vida percebida na carreira docente em educação física. Rev Bras Educ Fís Esp. 2007;21(2):81-93.

LINDH-ASTRAND L, HOFFMANN M, HAMMAR M, KJELLGREN KI. Women's conception of the menopausal transition-a qualitative study. J Clin Nurs. 2007;16(3):509-17.

MADUREIRA AS, FONSECA AS, MAIA MFM. Estilo de vida e atividade física habitual de professores de Educação Física. Rev Bras Cineantropom Desempenho Hum. 2003;5(1):54-62.

MELEIRO AMAS. O stress do professor. In: Lipp M. (Org.). 0 stress do professor. 5 ed. Campinas: Papirus; 2006. p. 11-27.

MONTEIRO E, MARIN C. Alimentos funcionais. Rev Bras Med. 2010;67(1):10-9

MORAES FP, COLLA LM. Alimentos funcionais e nutracêuticos: definições, legislação e benefícios à saúde. Rev Eletr Farmácia 2006;3(2):99-112.

NAHAS MV, BARROS MVG, FRANCALACCI VL. O pentáculo do bem-estar: base conceitual para avaliação do estilo de vida de indivíduos e grupos. Rev Bras Ativ Fís Saúde 2000;5(2):48-59.

NAHAS MV. Atividade física, saúde e qualidade de vida: conceitos e sugestões para um estilo de vida ativo. 5 ed. Londrina: Midiograf, 2010.

OZEN AE, PONS A, TUR JA. Worldwide consumption of functional foods: a systematic review. Nutr Rev. 2014;70 (8): 472-481.

ROBERFROID M. Functional food concept and its application to prebiotics. Dig Liver Dis. 2002;34(2):105-10.

ROCHA MDHA, ROCHA PA. Do climatério à menopausa; Rev Científ ITPAC 2010;3(1):247.

RODRIGUEZ-AÑEZ CR, REIS RS, PETROSKI EL. Versão brasileira do questionário Estilo de Vida Fantástico: tradução e validação para adultos jovens. Arq Bras Cardiol. 2008;91(2):102-9. 
SALLES W, EGERLAND EM, BARROSO MLC, SOUZA CA. Estilo de vida e perfil socioeconômico de docentes dos cursos de educação física da Universidade Federal de Santa Catarina - UFSC. Rev Bras Ciên Saúde 2012;10(34):7-14.

SANTOS MN, MARQUES AC. Condições de saúde, estilo de vida e características de trabalho de professores de uma cidade do sul do Brasil. Ciên Saúde Colet. 2013;18(3):837-46.

SILVA MEP. Burnout: por que sofrem os professores? Est Pesq Psicologia 2006;6(1):8998.

SILVA-FILHO EA, COSTA AM. Avaliação da qualidade de vida de mulheres no climatério atendidas em hospital-escola na cidade do Recife, Brasil. Rev Bras Ginecol Obstet. 2008;30(3):113-20.

SOUZA PHM, SOUZA NETO MH, MAIA GA. Componentes funcionais nos alimentos. Bol SBCTA 2003;37(2):127-35.

SZAKÁLY Z, SZENTE V, KÖVÉR G, POLERECZKI Z, SZIGETI O. The influence of lifestyle on health behavior and preference for functional foods. Appetite 2012;58(1):406-413.

TRIOLA MF. Introdução à estatística - Atualização da Tecnologia. 11 ed. Rio de Janeiro: Grupo Editorial Nacional - Livros Técnicos e Científicos, 2013.

VIDAL A, DIAS D, MARTINS E, OLIVEIRA R, NASCIMENTO R, CORREIA M. A ingestão de alimentos funcionais e sua contribuição para a diminuição da incidência de doenças. Cad Grad Ciênc Biol Saúde 2012;1(15):43-52.

WORLD HEALTH ORGANIZATION (WHO). The World health report 1998 - Life in the 21st century: a vision for all. Genebra: WHO, 1998. 


\section{ANEXO A - Questionário de Consumo Alimentar de Alimentos Funcionais - QCAAF}

1. Você sabe o que é um alimento funcional?

( ) $\operatorname{Sim}($ ) Não

2. Marque os alimentos que você ingere regularmente.

\begin{tabular}{|c|c|c|}
\hline \multicolumn{2}{|c|}{ CLASSE DE ALIMENTOS FUNCIONAIS } & EXEMPLOS \\
\hline$(\quad)$ & SOFLAVONAS & Soja e derivados. \\
\hline$(\quad)$ & ÔMEGA 3 & $\begin{array}{l}\text { Peixes marinhos como sardinha, salmão, atum, anchova, } \\
\text { arenque. }\end{array}$ \\
\hline$(\quad)$ & ÁCIDO LINOLÊNICO & Óleos de linhaça, soja, nozes e amêndoas. \\
\hline() & CATEQUINAS & $\begin{array}{l}\text { Chá verde, cerejas, amoras, framboesas, mirtilo, uva roxa, } \\
\text { vinho tinto. }\end{array}$ \\
\hline$(\quad)$ & LICOPENO & $\begin{array}{l}\text { Tomate e derivados, goiaba vermelha, pimentão } \\
\text { vermelho, melancia. }\end{array}$ \\
\hline$(\quad)$ & LUTEÍNA E ZEAXANTINA & Folhas verdes (luteína), pequi e milho (zeaxantina). \\
\hline$(\quad)$ & INDÓIS E ISOITICIANATOS & $\begin{array}{l}\text { Couve flor, repolho, brócolis, couve de bruxelas, rabanete, } \\
\text { mostarda. }\end{array}$ \\
\hline$(\quad)$ & FLAVONÓIDES & Soja, frutas cítricas, tomate, pimentão, alcachofra, cereja. \\
\hline$(\quad)$ & FIBRAS SOLÚVEIS E INSOLÚVEIS & $\begin{array}{l}\text { Cereais integrais como aveia, centeio, cevada, farelo de } \\
\text { trigo, etc; Leguminosas como soja, feijão, ervilha, } \\
\text { hortaliças com talos e frutas com casca. }\end{array}$ \\
\hline$(\quad)$ & $\begin{array}{l}\text { PRÉBIÓTICOS } \\
\text { FRUTOOLIGOSSACARÍDEOS } \\
\text { INULINA }\end{array}$ & Extraídos de vegetais como raiz de chicória e batata yacon. \\
\hline$(\quad)$ & $\begin{array}{ll}\text { SULFETOS } & \text { ALÍLICOS } \\
\text { (ALILSULFETOS) } & \end{array}$ & Alho e cebola. \\
\hline$(\quad)$ & LIGNANAS & Linhaça, noz moscada. \\
\hline$(\quad)$ & TANINO & Maçã, sorgo, manjericão, manjerona, sálvia, uva, caju, soja. \\
\hline$(\quad)$ & ESTANÓIS E ESTERÓIS VEGETAIS & Extraídos de óleos vegetais como soja e de madeiras. \\
\hline() & $\begin{array}{l}\text { PROBIÓTICOS BÍFIDOBACTERIAS } \\
\text { LACTOBACILOS }\end{array}$ & $\begin{array}{l}\text { Leites fermentados, iogurtes e outros produtos lácteos } \\
\text { fermentados. }\end{array}$ \\
\hline$(\quad)$ & BETA CAROTENO & Manga, mamão, abóbora, cenoura. \\
\hline
\end{tabular}

